\title{
Quadrupole Hyperfine Structure in the Rotational Spectra of 1,2- and 1,3-Dichlorobenzene
}

\author{
I. Merke, Ch. Keussen, and H. Dreizler \\ Abteilung Chemische Physik im Institut für Physikalische Chemie der Universität Kiel \\ M. Onda \\ Department of Chemistry, Faculty of Science and Technology, Sophia University, Tokyo 102, Japan
}

Z. Naturforsch. 45a, 1273-1280 (1990); received August 16, 1990

The high resolution of microwave Fourier transform spectroscopy was used to investigate the ${ }^{35}$ chlorine hyperfine structure of 1,2 - and 1,3 -dichlorobenzene, $\mathrm{C}_{6} \mathrm{H}_{4}{ }^{35} \mathrm{Cl}_{2}$, very accurately in the rotational spectrum of their ground vibrational state. The careful analysis with a new evaluation program also yielded the off-diagonal element of the coupling tensor. The tensor elements are $\chi_{a a}=-41.1153(35) \mathrm{MHz}, \chi_{b b}=8.3415(96) \mathrm{MHz}, \chi_{c c}=32.7738(61) \mathrm{MHz}$, and $\chi_{a b}= \pm 52.41(58) \mathrm{MHz}$ for 1,2-dichlorobenzene and $\chi_{a a}=-44.174(12) \mathrm{MHz}, \chi_{b b}=10.876(12) \mathrm{MHz}, \chi_{c c}=33.298(12) \mathrm{MHz}$, and $\chi_{a b}= \pm 48.181$ (39) $\mathrm{MHz}$ for 1,3-dichlorobenzene.

\section{Introduction}

The microwave spectrum of 1,2-dichlorobenzene (1,2-DCB) was first measured and assigned by Onda et al. [1]. They determined the quadrupole coupling constants $\chi_{a a}, \chi_{b b}$, and $\chi_{c c}$ by an analysis of the envelope of the hyperfine (hfs) pattern and discussed the molecular structure, which was reinvestigated by Onda et al. $[2,3]$ analysing the spectra of six monodeuterated 1,2-DCB-3d and $-4 \mathrm{~d}$. They found the two $\mathrm{C}-\mathrm{Cl}$ bonds to be bent outward from each other by about $1^{\circ}$ with respect to an $120^{\circ} \mathrm{CCCl}$ angle. The orientation of the principal coupling tensor axes $g=x, y, z$ was assumed with the help of a comparison with values of $\chi_{x x}, \chi_{y y}$, and $\chi_{z z}$ of $1,3-\mathrm{DCB}$. Onda et al. also investigated the rotational spectrum of 1,3-DCB for the first time [4]. Their purpose was to obtain information about the planarity of the molecule and the $\mathrm{Cl}-\mathrm{Cl}$ distance. The ${ }^{35} \mathrm{Cl}$-hfs was observed but not analysed. A later investigation [5] of some Q-branch transitions allowed a rough determination of the coupling constants. It followed a partial $r_{0}$ structure based on the spectra of monodeuterated isotopomers [2] and a complete molecular structure based on the data of different techniques [6]. The purpose of our investigation is to improve the accuracy of the coupling constants by analysis of highly resolved spectra and to determine the off-diagonal coupling tensor element

Reprint requests to Prof. Dr. H. Dreizler, Institut für Physikalische Chemie der Universität Kiel, Olshausenstr. 40, D-2300 Kiel, FRG. $\chi_{a b}$. So the orientation of the coupling tensor and its principal coupling elements can be determined without assumptions.

\section{Experimental}

We purchased 1,2- and 1,3-DCB from Aldrich, Steinheim, and used it without further purification. First measurements were made with waveguide microwave Fourier transform (MWFT) spectrometers in $\mathrm{X}-(8-12.4 \mathrm{GHz}), \mathrm{Ku}-(12.4-18 \mathrm{GHz})$, and $\mathrm{K}$-band $(18-26.4 \mathrm{GHz})[7-9]$. These measurements were supplemented with our pulsed molecular beam (MB) MWFT spectrometer in two versions [10].

The waveguide spectra were recorded at temperatures of $-10{ }^{\circ} \mathrm{C}(1,2-\mathrm{DCB})$ and -15 to $-30^{\circ} \mathrm{C}(1,3$ $\mathrm{DCB})$ and pressures from 2 to $5 \mathrm{mTorr}$ ( 0.3 to $0.7 \mathrm{~Pa}$ ) and 1 to $2.5 \mathrm{mTorr}(0.15$ to $0.35 \mathrm{~Pa})$, respectively. The MB spectra were made with a stagnation pressure of about 200 to 400 Torr $\left(2.7 \cdot 10^{4}\right.$ to $\left.5.4 \cdot 10^{4} \mathrm{~Pa}\right)$ and about $1 \%$ substance in argon.

To eliminate overlap effects [11] the frequencies of transitions in the waveguide MWFT spectra were determined by a least squares fit of the time domain signal [12].

The line frequencies of the MB spectra were taken without additional treatment. These MB measurements were especially useful and necessary as the cooling effect eliminates spectra of excited vibrational states and high $J$ transitions. So the patterns were 
Table 1. Rotational transitions of 1,2-dichlorobenzene. $v$ : measured frequency $[\mathrm{MHz}], \delta_{\mathrm{hfs}}$ : difference between observed and calculated frequency of the hyperfine component $[\mathrm{kHz}], v_{0}$ : hypothetical unsplit line frequeny [MHz]. Measurements excluded from the fit of the quadrupole coupling constants because of limitations in computer memory and time are marked with an asterisk.

\begin{tabular}{|c|c|c|c|c|c|c|c|c|c|c|c|c|}
\hline $\mathrm{J}^{\prime}$ & $K_{-}^{\prime}$ & $\mathrm{K}_{+}^{\prime}$ & $+J$ & $\mathrm{~K}_{-}$ & $K_{+}$ & $F^{\prime}$ & $I^{\prime}$ & $\mathrm{F}$ & I & $v$ & $\delta_{\mathrm{hfs}}$ & $v_{0}$ \\
\hline 3 & 2 & 1 & +2 & 2 & 0 & $\begin{array}{l}1.0 \\
3.0 \\
3.0 \\
5.0 \\
6.0 \\
4.0 \\
3.0 \\
4.0 \\
5.0 \\
4.0 \\
2.0 \\
5.0 \\
3.0 \\
4.0 \\
2.0 \\
3.0\end{array}$ & $\begin{array}{l}3.0 \\
1.0 \\
2.0 \\
3.0 \\
3.0 \\
1.0 \\
3.0 \\
2.0 \\
2.0 \\
3.0 \\
1.0 \\
3.0 \\
0.0 \\
3.0 \\
2.0 \\
3.0\end{array}$ & $\begin{array}{l}1.0 \\
2.0 \\
2.0 \\
5.0 \\
5.0 \\
3.0 \\
2.0 \\
3.0 \\
4.0 \\
4.0 \\
1.0 \\
4.0 \\
2.0 \\
3.0 \\
2.0 \\
3.0\end{array}$ & $\begin{array}{l}3.0 \\
1.0 \\
0.0 \\
3.0 \\
3.0 \\
1.0 \\
3.0 \\
2.0 \\
2.0 \\
3.0 \\
1.0 \\
3.0 \\
2.0 \\
3.0 \\
2.0 \\
3.0\end{array}$ & $\begin{array}{l}7704.722 \\
7702.255 \\
7701.522 \\
7698.045 \\
7696.700 \\
7694.220 \\
7691.879 \\
7691.331 \\
7691.283 \\
7683.533 \\
7683.421 \\
7682.947 \\
7681.106 \\
7680.532 \\
7680.005 \\
7679.807\end{array}$ & $\begin{array}{r}-4 \\
2 \\
0 \\
-3 \\
-1 \\
-1 \\
-2 \\
7 \\
-2 \\
-2 \\
2 \\
2 \\
1 \\
0 \\
0 \\
-3\end{array}$ & 7691.347 \\
\hline 4 & 2 & 3 & +3 & 2 & 2 & $\begin{array}{l}1.0 \\
1.0 \\
4.0 \\
4.0 \\
2.0 \\
2.0 \\
7.0 \\
3.0 \\
3.0 \\
5.0 \\
6.0 \\
6.0 \\
4.0 \\
3.0 \\
4.0 \\
5.0\end{array}$ & $\begin{array}{l}3.0 \\
3.0 \\
1.0 \\
0.0 \\
3.0 \\
3.0 \\
3.0 \\
3.0 \\
3.0 \\
1.0 \\
2.0 \\
3.0 \\
3.0 \\
1.0 \\
2.0 \\
3.0\end{array}$ & $\begin{array}{l}0.0 \\
1.0 \\
3.0 \\
3.0 \\
1.0 \\
2.0 \\
6.0 \\
2.0 \\
3.0 \\
4.0 \\
5.0 \\
5.0 \\
3.0 \\
2.0 \\
3.0 \\
4.0\end{array}$ & $\begin{array}{l}3.0 \\
3.0 \\
1.0 \\
0.0 \\
3.0 \\
3.0 \\
3.0 \\
3.0 \\
3.0 \\
3.0 \\
2.0 \\
3.0 \\
3.0 \\
1.0 \\
2.0 \\
1.0\end{array}$ & $\begin{array}{l}8754.859 \\
8754.822 \\
8753.122 \\
8753.084 \\
8752.427 \\
8752.388 \\
8751.884 \\
8750.099 \\
8750.166 \\
8749.267 \\
8748.877 \\
8746.591 \\
8745.915 \\
8745.875 \\
8744.671 \\
8743.870\end{array}$ & $\begin{array}{r}6 \\
-8 \\
10 \\
-8 \\
5 \\
-7 \\
2 \\
2 \\
1 \\
1 \\
-1 \\
3 \\
4 \\
-8 \\
4 \\
-2\end{array}$ & 8748.858 \\
\hline 4 & 3 & 1 & +3 & 3 & 0 & $\begin{array}{l}1.0 \\
2.0 \\
6.0 \\
1.0 \\
4.0 \\
4.0 \\
3.0 \\
7.0 \\
2.0 \\
5.0 \\
5.0 \\
6.0 \\
3.0 \\
4.0 \\
5.0 \\
6.0 \\
3.0 \\
3.0 \\
4.0 \\
5.0 \\
4.0\end{array}$ & $\begin{array}{l}3.0 \\
3.0 \\
3.0 \\
3.0 \\
1.0 \\
2.0 \\
3.0 \\
3.0 \\
3.0 \\
1.0 \\
2.0 \\
2.0 \\
2.0 \\
3.0 \\
3.0 \\
3.0 \\
1.0 \\
3.0 \\
0.0 \\
3.0 \\
3.0\end{array}$ & $\begin{array}{l}0.0 \\
1.0 \\
6.0 \\
1.0 \\
3.0 \\
3.0 \\
2.0 \\
6.0 \\
2.0 \\
4.0 \\
4.0 \\
5.0 \\
2.0 \\
3.0 \\
5.0 \\
5.0 \\
2.0 \\
3.0 \\
3.0 \\
4.0 \\
4.0\end{array}$ & $\begin{array}{l}3.0 \\
3.0 \\
3.0 \\
3.0 \\
1.0 \\
2.0 \\
3.0 \\
3.0 \\
3.0 \\
1.0 \\
2.0 \\
2.0 \\
2.0 \\
3.0 \\
3.0 \\
3.0 \\
1.0 \\
3.0 \\
0.0 \\
3.0 \\
3.0\end{array}$ & $\begin{array}{r}10001.907 \\
9998.834 \\
9997.120 \\
9996.779 \\
9996.254 \\
9996.129 \\
9992.981 \\
9992.521 \\
9991.008 \\
9988.138 \\
9986.600 \\
9986.553 \\
9986.508 \\
9982.651 \\
9982.420 \\
9980.017 \\
9979.959 \\
9978.993 \\
9977.067 \\
9975.458 \\
9973.647\end{array}$ & $\begin{array}{r}-0 \\
3 \\
-3 \\
-1 \\
1 \\
3 \\
3 \\
0 \\
2 \\
0 \\
8 \\
-2 \\
-9 \\
0 \\
-1 \\
4 \\
-2 \\
-3 \\
2 \\
1 \\
-3\end{array}$ & 9986.614 \\
\hline
\end{tabular}

Table 1 (continued)

\begin{tabular}{|c|c|c|c|c|c|c|c|c|c|c|c|c|}
\hline $\mathrm{J}^{\mathrm{s}}$ & $\mathrm{K}$ : & $\mathrm{K}_{+}^{\prime}-$ & $-\mathrm{J}$ & $\mathrm{K}_{-}$ & $\mathrm{K}_{+}$ & $F^{\prime}$ & $I^{\prime}$ & F & I & $v$ & $\delta_{\mathrm{hfs}}$ & $v_{0}$ \\
\hline 5 & 1 & $5+$ & 4 & 1 & 4 & $\begin{array}{l}2.0 \\
3.0 \\
5.0 \\
8.0 \\
7.0 \\
5.0 \\
7.0 \\
2.0 \\
6.0 \\
6.0 \\
4.0 \\
4.0 \\
5.0 \\
3.0 \\
6.0 \\
7.0\end{array}$ & $\begin{array}{l}3.0 \\
3.0 \\
3.0 \\
3.0 \\
3.0 \\
1.0 \\
2.0 \\
3.0 \\
1.0 \\
3.0 \\
1.0 \\
3.0 \\
3.0 \\
3.0 \\
3.0 \\
3.0\end{array}$ & $\begin{array}{l}2.0 \\
3.0 \\
5.0 \\
7.0 \\
6.0 \\
4.0 \\
6.0 \\
1.0 \\
5.0 \\
5.0 \\
3.0 \\
3.0 \\
4.0 \\
2.0 \\
6.0 \\
7.0\end{array}$ & $\begin{array}{l}3.0 \\
3.0 \\
3.0 \\
3.0 \\
3.0 \\
1.0 \\
2.0 \\
3.0 \\
1.0 \\
3.0 \\
1.0 \\
3.0 \\
3.0 \\
3.0 \\
3.0 \\
3.0\end{array}$ & $\begin{array}{l}9041.725 \\
9039.819 \\
9039.221 \\
9036.059 \\
9035.670 \\
9035.611 \\
9034.987 \\
9034.776 \\
9034.702 \\
9034.301 \\
9034.148 \\
9033.619 \\
9033.213 \\
9033.159 \\
9026.346 \\
9020.336\end{array}$ & $\begin{array}{r}-5 \\
1 \\
2 \\
3 \\
8 \\
-4 \\
-2 \\
-5 \\
0 \\
-2 \\
2 \\
2 \\
1 \\
0 \\
-1 \\
1\end{array}$ & 9034.985 \\
\hline 6 & 1 & $5+$ & 5 & 1 & 4 & $\begin{array}{l}9.0 \\
6.0 \\
8.0 \\
4.0 \\
5.0 \\
5.0 \\
6.0 \\
7.0 \\
6.0\end{array}$ & $\begin{array}{l}3.0 \\
1.0 \\
2.0 \\
3.0 \\
3.0 \\
1.0 \\
2.0 \\
3.0 \\
3.0\end{array}$ & $\begin{array}{l}8.0 \\
5.0 \\
7.0 \\
3.0 \\
4.0 \\
4.0 \\
5.0 \\
6.0 \\
5.0\end{array}$ & $\begin{array}{l}3.0 \\
1.0 \\
2.0 \\
3.0 \\
3.0 \\
1.0 \\
2.0 \\
3.0 \\
3.0\end{array}$ & $\begin{array}{l}12384.630 \\
12384.591 \\
12383.565 \\
12383.322 \\
12383.269 \\
12382.554 \\
12382.484 \\
12382.409 \\
12382.275\end{array}$ & $\begin{array}{r}7 \\
-5 \\
0 \\
6 \\
0 \\
4 \\
-16 \\
0 \\
5\end{array}$ & 12383.560 \\
\hline 6 & 2 & $5+$ & 5 & 2 & 4 & $\begin{array}{l}6.0 \\
9.0 \\
8.0 \\
5.0 \\
7.0 \\
4.0 \\
6.0\end{array}$ & $\begin{array}{l}0.0 \\
3.0 \\
2.0 \\
2.0 \\
2.0 \\
2.0 \\
2.0\end{array}$ & $\begin{array}{l}5.0 \\
8.0 \\
7.0 \\
4.0 \\
6.0 \\
3.0 \\
5.0\end{array}$ & $\begin{array}{l}0.0 \\
3.0 \\
2.0 \\
2.0 \\
2.0 \\
2.0 \\
2.0\end{array}$ & $\begin{array}{l}12301.552 \\
12301.482 \\
12300.169 \\
12300.169 \\
12300.169 \\
12300.169 \\
12298.796\end{array}$ & $\begin{array}{r}13 \\
9 \\
-8 \\
-3 \\
-4 \\
-8 \\
1\end{array}$ & 12300.168 \\
\hline 8 & 5 & $3+$ & 7 & 5 & 更 & $\begin{array}{r}5.0 \\
8.0 \\
11.0 \\
6.0 \\
7.0 \\
9.0 \\
10.0 \\
10.0 \\
7.0 \\
8.0 \\
8.0 \\
9.0\end{array}$ & $\begin{array}{l}3.0 \\
1.0 \\
3.0 \\
3.0 \\
3.0 \\
1.0 \\
2.0 \\
3.0 \\
1.0 \\
3.0 \\
0.0 \\
3.0\end{array}$ & $\begin{array}{r}4.0 \\
7.0 \\
10.0 \\
5.0 \\
6.0 \\
8.0 \\
9.0 \\
9.0 \\
6.0 \\
7.0 \\
7.0 \\
8.0\end{array}$ & $\begin{array}{l}3.0 \\
1.0 \\
3.0 \\
3.0 \\
3.0 \\
1.0 \\
2.0 \\
3.0 \\
1.0 \\
3.0 \\
0.0 \\
3.0\end{array}$ & $\begin{array}{l}20359.220 \\
20358.528 \\
20357.964 \\
20356.861 \\
20355.701 \\
20355.550 \\
20355.447 \\
20354.350 \\
20352.996 \\
20352.701 \\
20352.368 \\
20351.889\end{array}$ & $\begin{array}{r}-4 \\
-1 \\
4 \\
-5 \\
1 \\
-7 \\
8 \\
0 \\
0 \\
-5 \\
3 \\
6 \\
-2\end{array}$ & 20355.444 \\
\hline
\end{tabular}


Table 1 (continued)

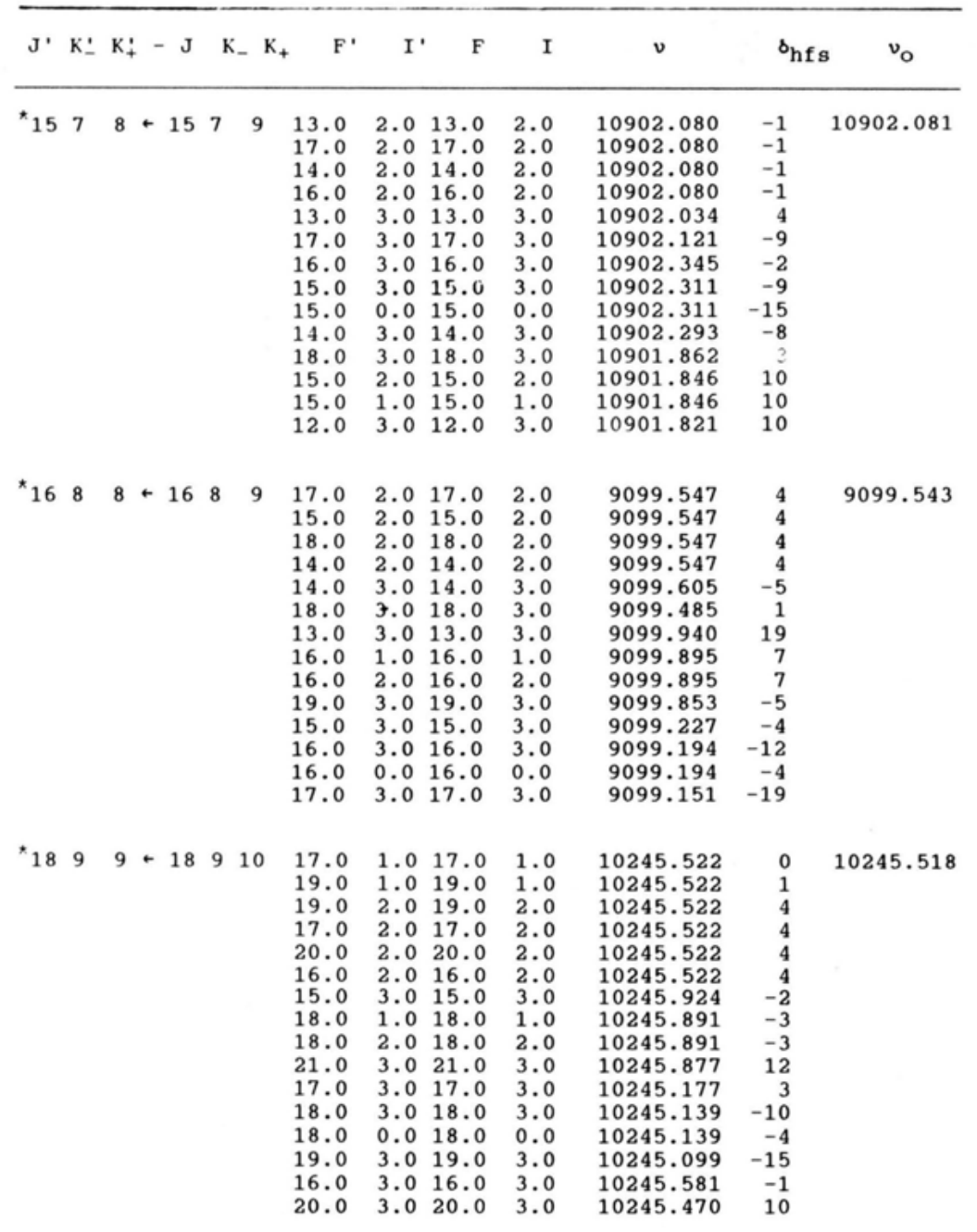

Table 1 (continued)

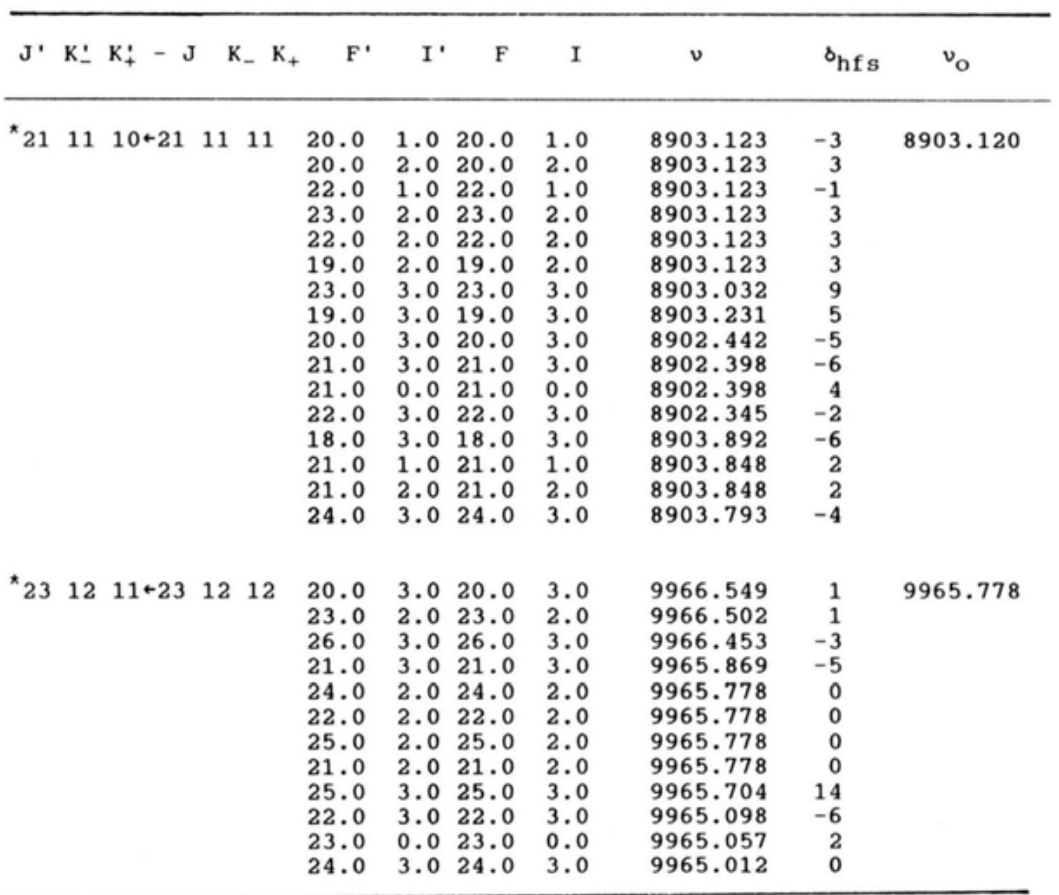


Table 2. Measured rotational transition frequencies of 1,3-dichlorobenzene. Explanation of symbols see Table 1.

\begin{tabular}{|c|c|c|c|c|c|c|c|c|c|c|c|c|}
\hline $\mathrm{J}^{\prime}$ & $\mathrm{K}:$ & $\mathrm{K}_{+}$ & $+\mathrm{J}$ & $\mathrm{K}_{-}$ & $\mathrm{K}_{+}$ & $F^{\prime}$ & $I^{\prime}$ & F & I & $v$ & $s_{\mathrm{hfs}}$ & $v_{0}$ \\
\hline 4 & 2 & 3 & $3 \cdot 3$ & 1 & 2 & $\begin{array}{l}4.0 \\
7.0 \\
3.0 \\
5.0 \\
5.0 \\
6.0 \\
4.0\end{array}$ & $\begin{array}{l}0.0 \\
3.0 \\
2.0 \\
1.0 \\
2.0 \\
2.0 \\
2.0\end{array}$ & $\begin{array}{l}3.0 \\
6.0 \\
2.0 \\
4.0 \\
4.0 \\
5.0 \\
3.0\end{array}$ & $\begin{array}{l}0.0 \\
3.0 \\
2.0 \\
1.0 \\
2.0 \\
2.0 \\
2.0\end{array}$ & $\begin{array}{l}11699.838 \\
11700.750 \\
11701.107 \\
11701.338 \\
11701.525 \\
11701.572 \\
11702.206\end{array}$ & $\begin{array}{r}-1 \\
-1 \\
2 \\
-2 \\
-4 \\
7 \\
0\end{array}$ & 11700.774 \\
\hline 4 & 4 & 1 & $1+3$ & 3 & 0 & $\begin{array}{l}2.0 \\
6.0 \\
2.0 \\
5.0 \\
3.0 \\
5.0 \\
6.0 \\
4.0 \\
7.0\end{array}$ & $\begin{array}{l}2.0 \\
2.0 \\
2.0 \\
2.0 \\
2.0 \\
2.0 \\
3.0 \\
2.0 \\
3.0\end{array}$ & $\begin{array}{l}2.0 \\
5.0 \\
1.0 \\
4.0 \\
2.0 \\
5.0 \\
5.0 \\
3.0 \\
6.0\end{array}$ & $\begin{array}{l}2.0 \\
2.0 \\
2.0 \\
2.0 \\
2.0 \\
2.0 \\
3.0 \\
2.0 \\
3.0\end{array}$ & $\begin{array}{l}20590.414 \\
20590.414 \\
20590.414 \\
20590.414 \\
20590.414 \\
20590.414 \\
20589.624 \\
20588.950 \\
20588.396\end{array}$ & $\begin{array}{r}3 \\
-3 \\
8 \\
-10 \\
1 \\
22 \\
-10 \\
1 \\
-7\end{array}$ & 20590.427 \\
\hline 4 & 4 & 0 & +3 & 3 & 1 & $\begin{array}{l}5.0 \\
6.0 \\
2.0 \\
5.0 \\
3.0 \\
6.0 \\
4.0 \\
4.0 \\
7.0 \\
3.0\end{array}$ & $\begin{array}{l}1.0 \\
2.0 \\
2.0 \\
2.0 \\
2.0 \\
3.0 \\
1.0 \\
2.0 \\
3.0 \\
3.0\end{array}$ & $\begin{array}{l}4.0 \\
5.0 \\
1.0 \\
4.0 \\
2.0 \\
5.0 \\
3.0 \\
3.0 \\
6.0 \\
3.0\end{array}$ & $\begin{array}{l}1.0 \\
2.0 \\
2.0 \\
2.0 \\
2.0 \\
3.0 \\
1.0 \\
2.0 \\
3.0 \\
3.0\end{array}$ & $\begin{array}{l}20591.688 \\
20591.106 \\
20591.106 \\
20591.106 \\
20591.106 \\
20590.313 \\
20589.725 \\
20589.615 \\
20589.085 \\
20579.300\end{array}$ & $\begin{array}{r}5 \\
6 \\
16 \\
-1 \\
10 \\
-9 \\
0 \\
-9 \\
5 \\
9\end{array}$ & 20591.112 \\
\hline 5 & 4 & 2 & $2+4$ & 3 & 1 & $\begin{array}{l}7.0 \\
6.0 \\
5.0 \\
4.0 \\
7.0 \\
5.0 \\
4.0 \\
7.0 \\
4.0 \\
6.0 \\
3.0 \\
8.0 \\
5.0 \\
2.0 \\
4.0 \\
2.0\end{array}$ & $\begin{array}{l}3.0 \\
3.0 \\
3.0 \\
1.0 \\
3.0 \\
3.0 \\
3.0 \\
2.0 \\
2.0 \\
2.0 \\
3.0 \\
3.0 \\
1.0 \\
3.0 \\
3.0 \\
3.0\end{array}$ & $\begin{array}{l}7.0 \\
5.0 \\
4.0 \\
3.0 \\
6.0 \\
5.0 \\
3.0 \\
6.0 \\
3.0 \\
5.0 \\
2.0 \\
7.0 \\
4.0 \\
1.0 \\
4.0 \\
2.0\end{array}$ & $\begin{array}{l}3.0 \\
3.0 \\
3.0 \\
1.0 \\
3.0 \\
3.0 \\
3.0 \\
2.0 \\
2.0 \\
2.0 \\
3.0 \\
3.0 \\
1.0 \\
3.0 \\
3.0 \\
3.0\end{array}$ & $\begin{array}{l}22121.768 \\
22118.830 \\
22118.233 \\
22117.742 \\
22116.565 \\
22116.223 \\
22115.660 \\
22115.654 \\
22115.654 \\
22115.654 \\
22114.630 \\
22113.410 \\
22112.959 \\
22112.461 \\
22111.552 \\
22110.125\end{array}$ & $\begin{array}{r}1 \\
-3 \\
-4 \\
-11 \\
-2 \\
-1 \\
-9 \\
10 \\
4 \\
8 \\
-5 \\
-3 \\
-1 \\
2 \\
-9 \\
2\end{array}$ & 22115.670 \\
\hline
\end{tabular}

Table 2 (continued)

\begin{tabular}{|c|c|c|c|c|c|c|c|c|c|c|c|c|}
\hline $\mathrm{J}^{\prime}$ & $\mathrm{K}:$ & $\mathrm{K}_{4}$ & $+\mathrm{J}$ & $\mathrm{K}_{-}$ & $\mathrm{K}_{+}$ & $F^{\prime}$ & $I^{\prime}$ & F & I & $v$ & $\delta_{\mathrm{hfs}}$ & $v_{0}$ \\
\hline 5 & 4 & 1 & +4 & 3 & 2 & $\begin{array}{l}5.0 \\
6.0 \\
5.0 \\
5.0 \\
4.0 \\
7.0 \\
4.0 \\
3.0 \\
3.0 \\
7.0 \\
4.0 \\
6.0 \\
6.0 \\
8.0 \\
5.0 \\
5.0\end{array}$ & $\begin{array}{l}0.0 \\
3.0 \\
0.0 \\
3.0 \\
1.0 \\
3.0 \\
3.0 \\
2.0 \\
2.0 \\
2.0 \\
2.0 \\
2.0 \\
2.0 \\
3.0 \\
2.0 \\
2.0\end{array}$ & $\begin{array}{l}5.0 \\
5.0 \\
4.0 \\
4.0 \\
3.0 \\
6.0 \\
3.0 \\
3.0 \\
2.0 \\
6.0 \\
3.0 \\
5.0 \\
6.0 \\
7.0 \\
4.0 \\
5.0\end{array}$ & $\begin{array}{l}2.0 \\
3.0 \\
0.0 \\
3.0 \\
1.0 \\
3.0 \\
3.0 \\
2.0 \\
2.0 \\
2.0 \\
2.0 \\
2.0 \\
2.0 \\
3.0 \\
2.0 \\
2.0\end{array}$ & $\begin{array}{l}22127.424 \\
22123.795 \\
22123.325 \\
22123.168 \\
22122.694 \\
22121.505 \\
22120.562 \\
22120.562 \\
22120.562 \\
22120.562 \\
22120.562 \\
22120.562 \\
22120.562 \\
22118.318 \\
22117.840 \\
22113.712\end{array}$ & $\begin{array}{r}-3 \\
7 \\
3 \\
-10 \\
-4 \\
5 \\
-11 \\
0 \\
16 \\
-3 \\
-4 \\
-4 \\
11 \\
11 \\
2 \\
-8\end{array}$ & 22120.586 \\
\hline 5 & 1 & 5 & +4 & 0 & 4 & $\begin{array}{l}6.0 \\
5.0 \\
3.0 \\
6.0 \\
4.0 \\
5.0 \\
4.0 \\
3.0 \\
7.0 \\
6.0 \\
7.0 \\
8.0 \\
5.0 \\
3.0\end{array}$ & $\begin{array}{l}3.0 \\
3.0 \\
3.0 \\
3.0 \\
3.0 \\
0.0 \\
1.0 \\
2.0 \\
2.0 \\
1.0 \\
3.0 \\
3.0 \\
3.0 \\
3.0\end{array}$ & $\begin{array}{l}6.0 \\
4.0 \\
2.0 \\
5.0 \\
3.0 \\
4.0 \\
3.0 \\
2.0 \\
6.0 \\
5.0 \\
6.0 \\
7.0 \\
5.0 \\
3.0\end{array}$ & $\begin{array}{l}3.0 \\
3.0 \\
3.0 \\
3.0 \\
3.0 \\
0.0 \\
1.0 \\
2.0 \\
2.0 \\
1.0 \\
3.0 \\
3.0 \\
3.0 \\
3.0\end{array}$ & $\begin{array}{l}8290.888 \\
8298.395 \\
8298.619 \\
8298.817 \\
8299.050 \\
8299.127 \\
8299.295 \\
8299.832 \\
8300.111 \\
8300.264 \\
8301.096 \\
8301.792 \\
8304.385 \\
8305.294\end{array}$ & $\begin{array}{r}-2 \\
2 \\
-1 \\
1 \\
0 \\
-3 \\
0 \\
0 \\
-5 \\
1 \\
1 \\
1 \\
-1 \\
2 \\
4\end{array}$ & 8300.529 \\
\hline 6 & 3 & 4 & +6 & 2 & 5 & $\begin{array}{l}6.0 \\
9.0 \\
5.0 \\
8.0 \\
8.0 \\
7.0 \\
7.0 \\
4.0 \\
6.0 \\
5.0\end{array}$ & $\begin{array}{l}2.0 \\
3.0 \\
2.0 \\
3.0 \\
2.0 \\
2.0 \\
1.0 \\
2.0 \\
0.0 \\
1.0\end{array}$ & $\begin{array}{l}6.0 \\
9.0 \\
5.0 \\
8.0 \\
8.0 \\
7.0 \\
7.0 \\
4.0 \\
6.0 \\
5.0\end{array}$ & $\begin{array}{l}2.0 \\
3.0 \\
2.0 \\
3.0 \\
2.0 \\
2.0 \\
1.0 \\
2.0 \\
0.0 \\
1.0\end{array}$ & $\begin{array}{l}10601.356 \\
10603.124 \\
10605.483 \\
10607.046 \\
10607.367 \\
10607.784 \\
10608.107 \\
10609.578 \\
10610.362 \\
10610.966\end{array}$ & $\begin{array}{r}3 \\
-2 \\
-1 \\
-2 \\
-1 \\
1 \\
-1 \\
-3 \\
-2 \\
2\end{array}$ & 10604.960 \\
\hline${ }^{\star} 13$ & 3 & 10 & +13 & 2 & 11 & $\begin{array}{l}13.0 \\
11.0 \\
15.0 \\
12.0 \\
14.0 \\
13.0\end{array}$ & $\begin{array}{l}0.0 \\
2.0 \\
2.0 \\
2.0 \\
2.0 \\
2.0\end{array}$ & $\begin{array}{l}13.0 \\
11.0 \\
15.0 \\
12.0 \\
14.0 \\
13.0\end{array}$ & $\begin{array}{l}0.0 \\
2.0 \\
2.0 \\
2.0 \\
2.0 \\
2.0\end{array}$ & $\begin{array}{l}8029.838 \\
8028.694 \\
8028.694 \\
8028.694 \\
8028.694 \\
8027.564\end{array}$ & $\begin{array}{r}9 \\
-2 \\
-2 \\
-2 \\
-2 \\
1\end{array}$ & 8028.696 \\
\hline
\end{tabular}




\section{Table 2 (continued)}

\begin{tabular}{|c|c|c|c|c|c|c|c|c|c|c|c|}
\hline $\mathrm{J}^{\prime} \quad \mathrm{K}$ - & $\mathrm{K}_{+}$ & $-\mathrm{J}$ & $\mathrm{K}_{-}$ & $\mathrm{K}_{+}$ & $F^{\prime}$ & $I^{\prime}$ & F & I & $v$ & $\delta_{\mathrm{hfs}}$ & $v_{0}$ \\
\hline${ }^{\star} 132$ & 11 & +12 & 31 & 10 & $\begin{array}{l}13.0 \\
11.0 \\
15.0 \\
12.0 \\
14.0 \\
13.0\end{array}$ & $\begin{array}{l}0.0 \\
2.0 \\
2.0 \\
2.0 \\
2.0 \\
2.0\end{array}$ & $\begin{array}{l}12.0 \\
10.0 \\
14.0 \\
11.0 \\
13.0 \\
12.0\end{array}$ & $\begin{array}{l}0.0 \\
2.0 \\
2.0 \\
2.0 \\
2.0 \\
2.0\end{array}$ & $\begin{array}{l}15371.648 \\
15371.095 \\
15371.095 \\
15371.095 \\
15371.095 \\
15370.539\end{array}$ & $\begin{array}{r}6 \\
1 \\
1 \\
1 \\
1 \\
-8\end{array}$ & 15371.095 \\
\hline *14 3 & 11 & +14 & 21 & 12 & $\begin{array}{l}15.0 \\
14.0 \\
13.0 \\
16.0 \\
15.0 \\
13.0 \\
12.0 \\
17.0 \\
14.0 \\
11.0\end{array}$ & $\begin{array}{l}3.0 \\
3.0 \\
1.0 \\
3.0 \\
1.0 \\
3.0 \\
3.0 \\
3.0 \\
1.0 \\
3.0\end{array}$ & $\begin{array}{l}15.0 \\
14.0 \\
13.0 \\
16.0 \\
15.0 \\
13.0 \\
12.0 \\
17.0 \\
14.0 \\
11.0\end{array}$ & $\begin{array}{l}3.0 \\
3.0 \\
1.0 \\
3.0 \\
1.0 \\
3.0 \\
3.0 \\
3.0 \\
1.0 \\
3.0\end{array}$ & $\begin{array}{l}8468.816 \\
8468.645 \\
8468.532 \\
8467.532 \\
8467.254 \\
8467.254 \\
8466.953 \\
8465.993 \\
8465.857 \\
8465.696\end{array}$ & $\begin{array}{r}3 \\
3 \\
3 \\
-11 \\
1 \\
10 \\
-3 \\
-2 \\
1 \\
-6\end{array}$ & 8467.269 \\
\hline * 153 & 12 & +15 & 21 & 13 & $\begin{array}{l}15.0 \\
13.0 \\
17.0 \\
14.0 \\
16.0 \\
15.0\end{array}$ & $\begin{array}{l}0.0 \\
2.0 \\
2.0 \\
2.0 \\
2.0 \\
2.0\end{array}$ & $\begin{array}{l}15.0 \\
13.0 \\
17.0 \\
14.0 \\
16.0 \\
15.0\end{array}$ & $\begin{array}{l}0.0 \\
2.0 \\
2.0 \\
2.0 \\
2.0 \\
2.0\end{array}$ & $\begin{array}{l}9187.907 \\
9186.206 \\
9186.206 \\
9186.206 \\
9186.206 \\
9184.513\end{array}$ & $\begin{array}{l}7 \\
-1 \\
-1 \\
-1 \\
-1 \\
-2\end{array}$ & 9186.207 \\
\hline * 163 & 13 & +16 & 21 & 14 & $\begin{array}{l}17.0 \\
16.0 \\
15.0 \\
18.0 \\
17.0 \\
15.0 \\
14.0 \\
19.0 \\
16.0 \\
13.0\end{array}$ & $\begin{array}{l}3.0 \\
3.0 \\
3.0 \\
3.0 \\
1.0 \\
1.0 \\
3.0 \\
3.0 \\
1.0 \\
3.0\end{array}$ & $\begin{array}{l}17.0 \\
16.0 \\
15.0 \\
18.0 \\
17.0 \\
15.0 \\
14.0 \\
19.0 \\
16.0 \\
13.0\end{array}$ & $\begin{array}{l}3.0 \\
3.0 \\
3.0 \\
3.0 \\
1.0 \\
1.0 \\
3.0 \\
3.0 \\
1.0 \\
3.0\end{array}$ & $\begin{array}{l}10189.876 \\
10189.664 \\
10189.523 \\
10188.106 \\
10187.754 \\
10187.754 \\
10187.390 \\
10185.984 \\
10185.835 \\
10185.651\end{array}$ & $\begin{array}{r}-1 \\
-9 \\
-7 \\
2 \\
1 \\
9 \\
-5 \\
-11 \\
10 \\
11\end{array}$ & 10187.771 \\
\hline * 194 & 15 & +19 & 3 & 16 & $\begin{array}{l}19.0 \\
17.0 \\
21.0 \\
18.0 \\
20.0 \\
19.0\end{array}$ & $\begin{array}{l}0.0 \\
2.0 \\
2.0 \\
2.0 \\
2.0 \\
2.0\end{array}$ & $\begin{array}{l}19.0 \\
17.0 \\
21.0 \\
18.0 \\
20.0 \\
19.0\end{array}$ & $\begin{array}{l}0.0 \\
2.0 \\
2.0 \\
2.0 \\
2.0 \\
2.0\end{array}$ & $\begin{array}{l}10873.546 \\
10872.534 \\
10872.534 \\
10872.534 \\
10872.534 \\
10871.522\end{array}$ & $\begin{array}{l}0 \\
0 \\
0 \\
0 \\
0 \\
0\end{array}$ & 10872.534 \\
\hline
\end{tabular}

Table 2 (continued)

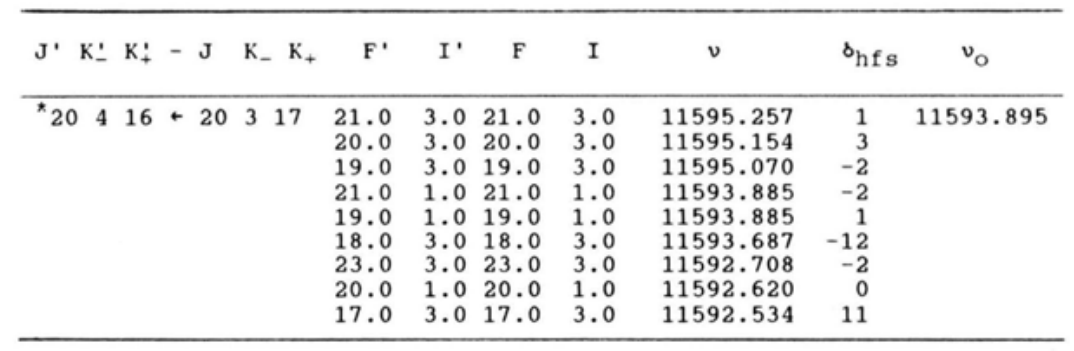

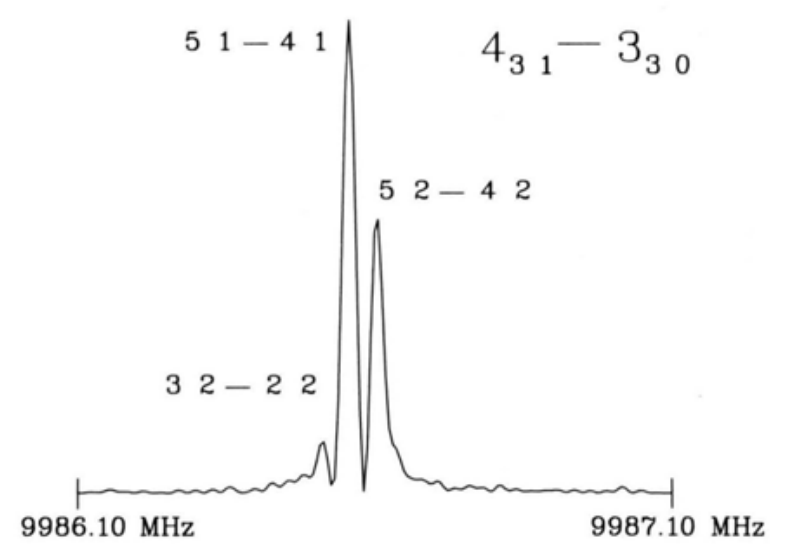

Fig. 1. A $1 \mathrm{MHz}$ scan of the rotational spectrum of 1,2-dichlorobenzene, showing part of the transition $J^{\prime} K_{-}^{\prime} K_{+}^{\prime} \leftarrow J K_{-} K_{+}=4_{31} \leftarrow 3_{30} . I$ and $F$ quantum num-
bers are given in the Figure. Polarizing frequency $9986.60 \mathrm{MHz}$, stagnation pressure 300 Torr $\left(4 \cdot 10^{4} \mathrm{~Pa}\right)$, ca. $1 \%$ substance in argon, 500 averaging cycles, sample interval $10 \mathrm{~ns}, 4096$ data points supplemented with 12288 zeros prior to Fourier transformation. 
cleared for overlaying lines and could be interpreted without ambiguity. In addition the accuracy of the frequencies increased. With this method complicated double resonance experiments could be avoided.

The measured frequencies are given in Table 1 for 1,2-DCB and Table 2 for 1,3-DCB.

The waveguide MWFT spectra are very crowded and the intensities are generally low.

So for 1,3-DCB as an example $1 \mathrm{R}$-branch and 6 Q-branch transitions with $J$ quantum numbers ranging from 12 to 20 could be recorded. They were insensitive to $\chi_{a b}$. With the MB MWFT measurements 1 Q-branch and 6 R-branch transitions could be assigned and resolved with $J$ ranging from 3 to 6 . Three of these transitions contain information on $\chi_{a b}$.

For 1,2-DCB the measurements revealed that some lines are split, which were calculated as singlets by a first order perturbation treatment (without $\chi_{a b}$ ). Figure 1 gives an example.

So the higher resolution urged for a more detailed analysis.

\section{Analysis}

One of us, I. Merke, wrote a program which sets up the Hamiltonian in a coupled basis. The coupling scheme $I_{1}+I_{2}=I, I+J=F$ is used. The Hamiltonian is based on considerations in [13]. Its matrix representation is diagonal in $F$. So $F$ submatrices are diagonalized. The program Q2DIG runs on the CRAY X-MP 216. Limitations on computer memory and time forced us to fit only transitions with low $J$ values. Fit parameters are $\chi_{a a}, \chi_{c c}$, and $\chi_{a b}$ and the hypothetical unsplit line frequencies or alternatively the rotational constants $A, B, C$ and the fourth order centrifugal distortion constants according to Watson's S-reduction.

We fitted the rotational and centrifugal distortion constants according to Watson's A-reduction in the $I^{r}$-representation with the program ZFAP4 [14] to all measured transitions. The centrifugal distortion constants are not well determined, but it was not our aim to determine them and their inclusion in the fit improves the reproduction of the spectra with the fitted constants.

The results are given in Table 3. It was possible to reproduce all measured hyperfine components within the measurement uncertainties.
Since the axis perpendicular to the molecular plane is a principal axis of inertia and coupling tensor ( $c$-and $y$-axis respectively), the knowledge of $\chi_{a b}$ allows the calculation of the angle $\Theta_{z a}$ between the $z$-principal axis of the coupling tensor and the $a$-principal axis of the inertia tensor and the $\chi_{g g}, g=x, y, z$, coupling elements in the principal axis system of the coupling tensor. For the axes systems see Fig. 2 and 3. The calculation is according to [15]. The results are also given in Table 3. For 1,2-DCB it should be noted, that a comparison with the structure [2] indicates that the $z$-axis of the coupling tensor is tilted by $1.5^{\circ}$ with

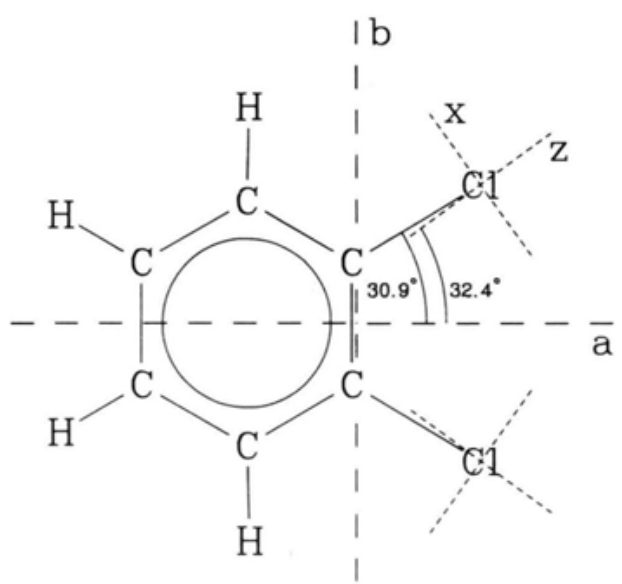

Fig. 2. 1,2-dichlorobenzene in its principal moment of inertia axis system $(a, b)$. The $x$ - and $z$-axes are principal axes of the quadrupole coupling tensor. The $z$-axis is tilted out of the $\mathrm{C}-\mathrm{Cl}$ internuclear axis by $1.5^{\circ}$. For better recognition this angle is drawn too large in the Figure.

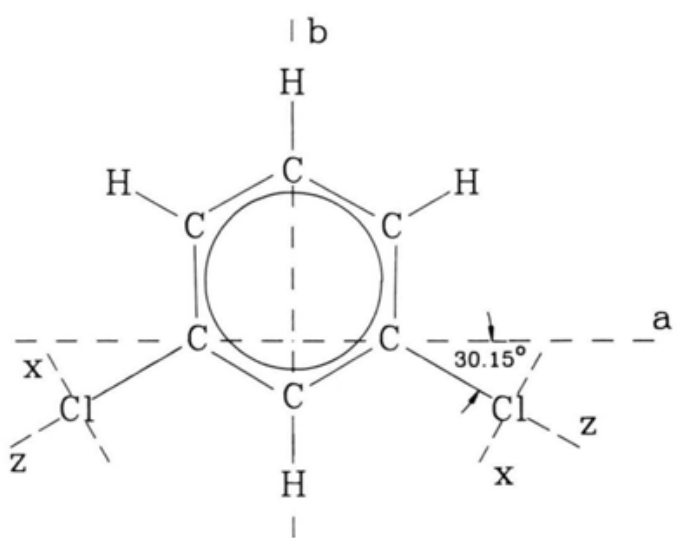

Fig. 3.1,3-dichlorobenzene in its principal moment of inertia axis system $(a, b)$. The $x$ - and $z$-axes are principal axes of the quadrupole coupling tensor. The $z$-axis coincidices with the $\mathrm{C}-\mathrm{Cl}$ bond axis. 
Table 3. Rotational, centrifugal distortion, and quadrupole coupling constants [MHz] of 1,2- and 1,3-dichlorobenzene. Rotational and centrifugal distortion constants determined from all measured transitions according to Watson's A-reduction, quadrupole coupling constants determined from the transitions not marked with an asterisk in Tables 1 and 2 (see text). Single standard errors in brackets. $\varkappa$ : Asymmetry parameter of the quadrupole coupling.

\begin{tabular}{|c|c|c|c|}
\hline \multicolumn{2}{|c|}{ Measured parameters } & \multicolumn{2}{|c|}{ Derived parameters } \\
\hline \multicolumn{4}{|c|}{ 1,2-Dichlorobenzene } \\
\hline $\begin{array}{l}A \\
B \\
C \\
\Delta_{J} \\
\Delta_{J K} \\
\Delta_{K} \\
\delta_{J} \\
\delta_{K} \\
\chi_{a a} \\
\chi_{c c} \\
\chi_{a b}\end{array}$ & $\begin{array}{c}1930.0814(40) \\
1431.1695(9) \\
821.6727(15) \\
3.2(18) \cdot 10^{-5} \\
8.7(66) \cdot 10^{-5} \\
26.4(82) \cdot 10^{-5} \\
1.99(91) \cdot 10^{-5} \\
5.0(78) \cdot 10^{-5} \\
-41.1153(35) \\
32.7738(61) \\
\pm 52.41(58)\end{array}$ & $\begin{array}{l}\chi_{b b} \\
\Theta \\
\chi_{x a} \\
\chi_{y y} \\
\chi_{z z} \\
x\end{array}$ & $\begin{array}{c}8.3415(96) \\
32.37(12)^{\circ} \\
41.57(52) \\
32.7738(61) \\
-74.34(52) \\
-0.1183\end{array}$ \\
\hline \multicolumn{4}{|c|}{ 1,3-dichlorobenzene } \\
\hline $\begin{array}{l}A \\
B \\
C \\
\Delta_{J} \\
\Delta_{J K} \\
\Delta_{K} \\
\delta_{J} \\
\delta_{K} \\
\chi_{a a} \\
\chi_{c c} \\
\chi_{a b}\end{array}$ & $\begin{array}{l}2832.3746(14) \\
862.8337(7) \\
661.2695(4) \\
2.23(18) \cdot 10^{-5} \\
-7.2(36) \cdot 10^{-5} \\
92.9(48) \cdot 10^{-5} \\
0.84(15) \cdot 10^{-5} \\
0.9(63) \cdot 10^{-5} \\
-44.174(12) \\
33.298(12) \\
\pm 48.181(39)\end{array}$ & $\begin{array}{l}\chi_{b b} \\
\Theta_{z a} \\
\chi_{x x} \\
\chi_{y y} \\
\chi_{z z} \\
x\end{array}$ & $\begin{array}{c}10.876(12) \\
30.13(1)^{\circ} \\
38.840(41) \\
33.298(12) \\
-72.138(41) \\
-0.0768\end{array}$ \\
\hline
\end{tabular}

respect to the $\mathrm{C}-\mathrm{Cl}$ bond direction. This supports the hypothesis of a bent bond between the carbon and chlorine. For 1,3-DCB the tilt angle is below $0.01^{\circ}$.

\section{Discussion}

In Table 4 we give a comparison of investigated ${ }^{35} \mathrm{Cl}$ principal axis coupling constants of chlorobenzenes. They vary in a range of $6 \%$ for $\chi_{z z}$ and $10 \%$ for $\chi_{y y}$, which is independent from a rotation transition. Most values differ beyond the standard error limits.

To compare the results with the quantum chemical theory we made $a b$ initio calculations with the Gaussian 86 program (G 86) [16] using the experimental structures of the dichlorobenzenes $[3,6]$ and chlorobenzene [2]. We included chlorobenzene [17] in our calculations to see which effects occur due to the second chlorine atom in the dichlorobenzenes. Because
Table 4. Electric field gradients $q_{g g^{\prime}}$ of chlorobenzene, 1,2and 1,3-dichlorobenzene calculated with Gaussian 86 (G 86) in the principal inertia axis system $\left(q_{g g^{\prime}}, g, g^{\prime}=a, b, c\right)$ and in the principal coupling tensor axis system $\left(q_{g g^{\prime}}, g=x, y, z\right)$. From the scale factors $f=\chi_{g g^{\prime}}(\exp ) / q_{g g^{\prime}}(\mathrm{G} 86)$ an averaged scale factor $f$ (standard error in brackets) is calculated, which in return is used to try to reproduce the measured $\chi_{g g^{\prime}}(\exp )$ following the formula $\chi_{g g^{\prime}}(\mathrm{G} 86)=\bar{f} \cdot q_{g g^{\prime}}(\mathrm{G} 86)$. Explanation of $\Theta_{z a}$ see text.

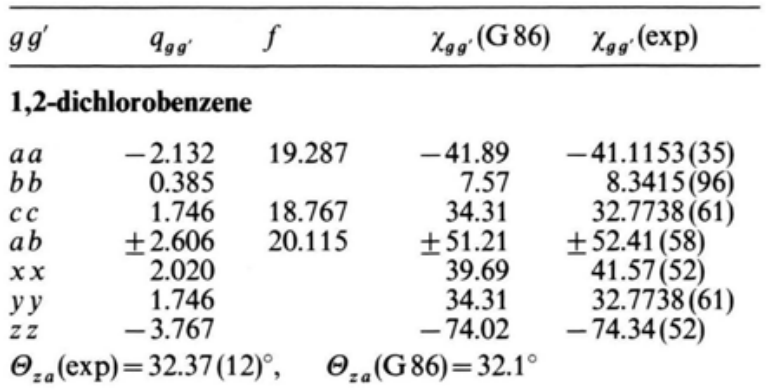

\section{1,3-dichlorobenzene}

$\begin{array}{lrrrr}a a & -2.264 & 19.514 & -44.49 & -44.174(12) \\ b b & 0.519 & & 10.20 & 10.876(12) \\ c c & 1.745 & 19.081 & 34.29 & 33.298(12) \\ a b & \pm 2.403 & 20.053 & \pm 47.22 & \pm 48.181(39) \\ x x & 1.903 & & 37.39 & 38.840(41) \\ y y & 1.745 & & 34.29 & 33.298(12) \\ z z & -3.649 & & -71.70 & -72.138(41) \\ \Theta_{z a}(\exp )=30.13(1)^{\circ}, \quad \Theta_{z a}(\mathrm{G} 86)=30.0^{\circ} & \end{array}$

\section{Chlorobenzene}

$\begin{array}{lrrrr}a a & -3.587 & 19.859 & -70.48 & -71.241(7) \\ b b & 1.867 & 20.484 & 36.69 & 38.237(10) \\ c c & 1.721 & & 33.82 & 33.005(10)\end{array}$

Average scale factor from all three molecules: $\bar{f}=19.65(50)$

of the limited computer memory we chose the relatively small $6-31 \mathrm{G}^{*}$ basis set [18] and did not take electron correlation into account. To get an impression of the quality of this basis set used for the calculation of the electric field gradients $g_{g g^{\prime}}\left(g, g^{\prime}=a, b, c\right.$ or $x, y, z$ ), we calculated an average scale factor $\bar{f}$ from all $f=\chi_{g g^{\prime}}(\exp ) / q_{g g^{\prime}}(\mathrm{G} 86)$, which correlates the measured $\chi_{g g^{\prime}}$ with the calculated electric field gradients. With an ideal basis set $f$ would be the same for all molecules and $g g^{\prime}$ combinations. As can be seen from Table 4, this averaged scale factor $\bar{f}$ allows to reproduce our experimental $\chi_{g g^{\prime}}$ within $7 \%$.

The calculation of the angle $\Theta_{z a}(\mathrm{G} 86)$ between the $z$-axis of the quadrupole coupling tensor and the $a$-axis of the principal inertia system of the molecules is independent of a scale factor. For 1,3-DCB the $z$-axis of the experimentally derived quadrupole tensor coincides with the $\mathrm{C}-\mathrm{Cl}$ bond direction. This coincidence is also the result of our quantum chemical calculations 
(see Table 4). In 1,2-DCB we found from the spectral analysis that the $z$-axis of the quadrupole tensor is tilted $1.5^{\circ}$ out of $\mathrm{C}-\mathrm{Cl}$ internuclear axis, which supports the hypothesis of a bent bond. The quantum chemical calculations were done with the experimental structure, which has an angle of $30.9^{\circ}$ between the $\mathrm{C}-\mathrm{Cl}$ internuclear axis and the $a$-axis. The calculated value for $\Theta_{z a}(\mathrm{G} 86)$ is $32.1^{\circ}$. This corresponds to a tilt angle of $1.2^{\circ}$ and a prediction of a bent bond, which is in reasonable agreement with the experiment. Perhaps the inclusion of electron correlation can still improve the calculations.

[1] M. Onda and I. Yamaguchi, J. Mol. Struct. 34, 1 (1976).

[2] M. Onda, Nippon Kagaku Kaishi 1986, 1476.

[3] M. Onda, M. Ueda, M. Atsuki, J. Yamaguchi, and I. Yamaguichi, J. Mol. Struct. 147, 77 (1986).

[4] M. Onda, O. Ohashi, and I. Yamaguchi, J. Mol. Struct. 31, 203 (1976).

[5] M. Onda and I. Yamaguchi, J. Mol. Struct. 36, 158 (1977).

[6] G. Anderson, S. Cradock, P. B. Liescheski, and D. W. H. Rankin, J. Mol. Struct. 216, 181 (1990).

[7] G. Bestmann and H. Dreizler, Z. Naturforsch. 37 a, 58 (1982).

[8] G. Bestmann, H. Dreizler, H. Mäder, and U. Andresen, Z. Naturforsch. 35a, 392 (1980).

[9] W. Stahl, G. Bestmann, H. Dreizler, U. Andresen, and R. Schwarz, Rev. Sci. Instrum. 56, 1759 (1985).

[10] U. Andresen, H. Dreizler, J. U. Grabow, and W. Stahl, to be published.

\section{Acknowledgement}

I. Merke, Ch. Keussen, and H. Dreizler thank the members of the Kiel group for help and discussion, especially $\mathrm{H}$. Krause for discussions about the Gaussian 86 program. Funds of the Deutsche Forschungsgemeinschaft, Fonds der Chemie, and the Land Schleswig-Holstein are gratefully acknowledged. The calculations were made at the computer center of the University of Kiel.

[11] I. Merke and H. Dreizler, Z. Naturforsch. 43a, 196 (1988).

[12] J. Haekel and H. Mäder, Z. Naturforsch. 43a, 203 (1988).

[13] W. Gordy and R. L. Cook, Microwave Molecular Spectra, J. Wiley, New York 1984, Chapter XV.

[14] Program ZFAP4, author V. Typke.

[15] W. Zeil, Fortschr. Chem. Forsch. 30, 114 (1972).

[16] Gaussian 86, M. J. Frisch, J. S. Binkley, H. B. Schlegel, K. Raghavachari, C. F. Melius, R. L. Martin, J. J. P. Stewart, F. W. Bobrowicz, C. M. Rohlfing, L. R. Kahn, D. J. Defrees, R. Seeger, R. A. Whiteside, D. J. Fox, E. M. Fleuder, and J. A. Pople, Carnegie-Mellon Quantum Chemistry Publishing Unit, Pittsburgh PA, 1984.

[17] N. Heineking, to be published.

[18] W. J. Hehre, R. Ditchfield, and J. A. Pople, J. Chem. Phys. 56, 2257 (1972). 